\section{Prevalência e fatores de risco para anemia em crianças pré-escolares do Estado de Alagoas, Brasil}

\section{The prevalence of and risk factors for anemia in preschool children in the State of Alagoas, in Brazil}

Regina Coeli da Silva Vieira 1

Haroldo da Silva Ferreira 2

Antonio Carlos Silva Costa 3

Fabiana Andréa Moura 4

Telma Maria de Menezes Toledo Florêncio 5

Zaira Maria Camerino Torres 6
1-6 Faculdade de Nutrição. Universidade Federal de Alagoas. Campus A.C. Simões, BR 104 Norte, Tabuleiro do Martins. Maceió, Alagoas, Brasil. CEP: 57.072-970.

E-mail: haroldo.ufal@gmail.com

\section{Abstract}

Objective: to estimate the prevalence and establish risk factors associated with anemia in children in the Brazilian State of Alagoas.

Methods: a cross-sectional study with a probabilistic sample of 666 children aged between 6 and 60 months. Anthropometric, environmental, demographic, socio-economic data were collected during home visits, along with information on health, the use of public services and the existence of diseases. The concentration of hemoglobin (Hb) was measured using a HemoCue ${ }^{\circledR}$ photometer, and a level of $<11$ $\mathrm{g} / \mathrm{dL}$ was taken to indicate anemia. Pearson's test was used identify correlations and the $\chi^{2}$ test to analyze associations between the category variables. The association of anemia with the predictor variables was confirmed by multiple logistic regression, with statistical significance set at $p<0.05$.

Results: the prevalence of anemia was $45.0 \% . \mathrm{Hb}$ levels were positively and significantly associated with age $(r=0.44 ; p<0.01)$. The highest prevalence was found in the 6-12 month age-group (75.2\%). Multivariate analysis identified the following variables associated with anemia: age $\leq 36$ months $(p<0.001)$ and a household with five or more occupants $(p=0.031)$.

Conclusions: the degree of prevalence of anemia found indicates that this is a serious public health problem in the State of Alagoas. Larger families and those with children aged under three years should receive greater attention.

Key words Anemia, Epidemiology, Child, Risk factors, Cross-sectional studies

\section{Resumo}

Objetivo: estimar a prevalência e os fatores de risco associados à anemia na população de pré-escolares do Estado de Alagoas, Brasil.

Métodos: estudo transversal com amostra probabilística de 666 crianças de 6 a 60 meses de idade. Por meio de inquérito domiciliar, realizado de novembro de 2005 a fevereiro de 2006, coletaram-se dados antropométricos, ambientais, demográficos, socioeconômicos, de saúde, de utilização de serviços públicos e a presença de morbidades. A concentração de hemoglobina ( $\mathrm{Hb}$ ) foi mensurada em fotometro HemoCue ${ }^{\circledR}$, considerando-se como anemia o nivel $<11$ g/dL. Para identificar correlações usou-se o teste de Pearson. O teste $\chi^{2}$ foi usado para analisar associações entre variáveis categóricas. A associação da anemia com as variáveis preditoras foi verificada por meio de análise de regressão logística múltipla. Adotou-se $p<0,05$ como nivel critico para definir significância estatística.

Resultados: a prevalência de anemia foi de 45, $0 \%$. O nivel de $\mathrm{Hb}$ se correlacionou de forma positiva e significativa à idade $(r=0,44 ; p<0,01)$. A prevalência máxima ocorreu na faixa etária de 6 a 12 meses $(75,2 \%)$. A análise multivariada identificou as seguintes variáveis associadas à anemia: idade da criança $\leq 36$ meses $(p<0,001)$ e domicílio com cinco ou mais pessoas $(p=0,031)$.

Conclusões: a prevalência de anemia apresentou magnitude que a caracteriza como grave problema de saúde pública em Alagoas. As famílias mais numerosas e com crianças menores de três anos são aquelas que devem receber maior nivel de atenção.

Palavras-chave Anemia, Epidemiologia, Criança, Fatores de risco, Estudos transversais 


\section{Introdução}

A anemia constitui um dos problemas nutricionais mais prevalentes do mundo. ${ }^{1}$ Estima-se que cerca de dois bilhões de pessoas possuem níveis de hemoglobina inferiores aos limiares considerados normais, condição que acarreta consequências deletérias à saúde e ao desenvolvimento infantil. ${ }^{2}$ Além da deficiência de ferro, que é a causa mais frequente de anemia, destacam-se outros fatores como as infecções agudas ou crônicas, outras deficiências de micronutrientes (especialmente folato, vitamina B12 e vitamina A) e doenças genéticas, tais como, a talassemia e a anemia falciforme. ${ }^{2}$ Estima-se que de $50 \%$ a $90 \%$ de todos os tipos de anemia no mundo ocorram pela deficiência de ferro. ${ }^{3}$

Embora a anemia seja um problema de distribuição global que atinge inclusive os países desenvolvidos, as condições socioeconômicas desfavoráveis representam um importante fator de risco. 4 No Brasil, é considerada o agravo nutricional de maior prevalência e cuja evolução, tem apresentado tendência positiva em pré-escolares.4-6

Na Pesquisa Nacional sobre Saúde e Nutrição (PNSN), realizada em 1989, o Estado de Alagoas, na Região Nordeste, apresentou as mais altas prevalências de desnutrição, com um percentual de $36,8 \%$ de crianças com déficit estatural, um valor bem mais elevado do que o observado no Estado de Santa Catarina (4,9\%), na Região Sul do país. ${ }^{7}$ Com base nesses dados, conclui-se que, tal como o déficit estatural, a anemia possivelmente represente um importante problema de saúde pública em pré-escolares neste Estado. Pesquisa realizada por Santos et al., 8 com 426 escolares da rede pública de Maceió, revelou uma prevalência de anemia em torno de $10 \%$. Outro levantamento realizado em préescolares da mesma cidade revelou níveis de prevalência de anemia próximos a 100\%.9 Apesar da possível gravidade do problema nessa população, a falta de dados oriundos de estudos de base populacional no Estado tem dificultado o planejamento e a avaliação de impacto de programas de prevenção e controle. Esse estudo tem por objetivo estimar a prevalência e investigar os fatores de risco associados à anemia na população em idade pré-escolar do Estado de Alagoas.

\section{Métodos}

Trata-se de um estudo transversal realizado em uma amostra representativa da população de crianças de 6 a 60 meses no Estado de Alagoas, Região Nordeste do Brasil, no período de novembro de 2005 a fevereiro de 2006.

O Estado possui cerca de três milhões de habitantes e caracteriza-se por apresentar os piores indicadores sociais no âmbito nacional ${ }^{10}$ sendo considerado o mais pobre do país, com renda per capita $52,6 \%$ abaixo da média brasileira, elevada concentração de renda, baixas coberturas de saneamento básico e elevados índices de analfabetismo. ${ }^{11}$

Para o cálculo da amostra adotou-se como parâmetros uma prevalência de 40,6\%, encontrada em crianças na mesma faixa etária no Estado de Pernambuco, 12 erro amostral de 3,5\% e um intervalo de confiança de $95 \%$. Considerando esses critérios, o tamanho da amostra calculado foi de 756 crianças.

O processo de amostragem foi em múltiplos estágios, em três etapas. $\mathrm{Na}$ primeira, foram sorteados 20 municípios por meio de amostragem sistemática com probabilidade proporcional ao tamanho (a cidade de Maceió, capital do Estado, foi sorteada seis vezes, haja vista que possui cerca de $1 / 3$ da população estadual). Na segunda etapa, quatro setores censitários foram sorteados dentro de cada município e, na terceira etapa, procedeu-se uma seleção sistemática dos domicílios dentro de cada setor, a partir do sorteio de um ponto inicial da contagem. Assim, em cada setor censitário, um certo número de domicílios eram visitados consecutivamente até a identificação de nove crianças na faixa etária estabelecida.

Os dados (demográficos, antropométricos, ambientais, socioeconômicos, de saúde, de utilização de serviços públicos e presença de morbidades) foram coletados por estudantes do curso de Nutrição utilizando formulário pré-testado em estudo piloto. As crianças e suas mães tiveram suas medidas de massa corporal e estatura aferidas. A massa corporal foi obtida em balança eletrônica portátil, com capacidade para $180 \mathrm{~kg}$ e sensibilidade para $100 \mathrm{~g}$ (Marte PP $\left.180^{\circledR}\right)$. O comprimento das crianças menores de dois anos foi verificado na posição de decúbito dorsal em estadiômetro pediátrico. As crianças com idade superior a dois anos e as suas respectivas mães foram medidas em posição ortostática, utilizando estadiômetro vertical. Ambos os equipamentos eram dotados de fita métrica inextensível com sensibilidade de $0,1 \mathrm{~cm}$.

A coleta da amostra de sangue (uma gota), por meio de punção da polpa digital, e a aferição da concentração de hemoglobina foram realizadas por uma auxiliar de enfermagem. Toda a equipe de campo foi devidamente treinada e o trabalho realizado sob supervisão.

A determinação dos níveis de hemoglobina $(\mathrm{Hb})$ foi realizada utilizando fotômetro HemoCue ${ }^{\circledR}$, teste 
diagnóstico considerado válido para estudos epidemiológicos.2,13 Consideraram-se anêmicas aquelas cuja concentração de $\mathrm{Hb}$ fosse inferior a $11 \mathrm{~g} / \mathrm{dL}, 2$ classificando-as da seguinte forma:14 anemia leve $(9,0<\mathrm{Hb}<11,0)$, anemia moderada $(7,0 \leq \mathrm{Hb} \leq 9,0)$ e anemia grave $(\mathrm{Hb}<7,0)$.

A avaliação socioeconômica, demográfica e ambiental foi procedida a partir do seguinte conjunto de variáveis: tipo de esgotamento sanitário, material predominante na construção da casa, número de cômodos da casa, acesso à água encanada, origem da água usada para beber, situação do domicílio, renda familiar total, renda per capita, número de membros da família, escolaridade materna, inscrição em programa assistencial do governo, sexo e posse de itens de consumo (televisão, fogão a gás, geladeira, máquina de lavar e automóvel), além de idade da mãe e da criança, idade em que a mãe teve o primeiro filho e a de ocorrência da menarca.

A avaliação das condições de saúde e utilização de serviços públicos foi realizada segundo a metodologia descrita por César et al.,15 tendo sido obtidas informações sobre problemas de saúde (diarréia e tosse) no momento da entrevista ou ocorridos nos últimos 15 dias, bem como sobre a ocorrência de problemas crônicos de saúde. Adicionalmente, obtiveram-se informações quanto à suplementação de vitamina A e internação hospitalar nos últimos 12 meses, realização de consulta pré-natal (sim ou não), utilização de creche e tipo de serviço de saúde utilizado.

As medidas das crianças foram combinadas para formar os índices peso-para-idade, estatura-paraidade e peso-para-altura, que foram expressos em escores Z. Para isso, utilizou-se o software Anthro, versão 2.0.2, que emprega o padrão antropométrico da World Health Organization-2006. A condição de déficit foi definida por $Z<-2$. O peso ao nascer (PN), obtido por observação direta no "cartão da criança", foi analisado de forma dicotômica: PN insuficiente $(<3000 \mathrm{~g})$ e PN adequado $(\geq 3000 \mathrm{~g})$. As medidas das mães com idades iguais ou superiores a 19 anos foram utilizadas para compor o Índice de Massa Corporal (IMC, $\mathrm{Kg} / \mathrm{m}^{2}$ ), adotando-se a seguinte classificação: Baixo peso $\left(<18,5 \mathrm{Kg} / \mathrm{m}^{2}\right)$, Normal ( $\left.\geq 18,5 \mathrm{a}<25 \mathrm{Kg} / \mathrm{m}^{2}\right)$, Sobrepeso ( $\geq 25 \mathrm{a}<30$ $\left.\mathrm{Kg} / \mathrm{m}^{2}\right)$ e Obesidade: $\left(\geq 30 \mathrm{Kg} / \mathrm{m}^{2}\right)$.

Para a análise estatística, os dados foram digitados em dupla entrada independente em formulário criado no Epi-info, versão 3.3.2. Após comparação dos arquivos e correção de divergências, procederam-se às análises com auxílio do pacote estatístico SPSS, versão 13.0.

$\mathrm{Na}$ análise bivariada foram empregados o teste
' $\mathrm{t}$ ', para a comparação entre duas médias de $\mathrm{Hb}$, ou ANOVA seguido do teste de Tukey, na comparação entre mais de dois grupos (variáveis contínuas). A indicação do uso de estatística paramétrica foi investigada por meio do teste de Kolmogorov-Smirnov e pelo teste de Levene. A associação entre anemia (variável categórica) e os fatores investigados foi testada pela aplicação do teste qui-quadrado. A magnitude da associação foi estimada pelo cálculo da razão de chances (RC) e seus respectivos intervalos de confiança de 95\% (IC95\%). O coeficiente de correlação de Pearson foi utilizado para testar correlações simples. As diferenças foram consideradas estatisticamente significativas quando $p<0,05$.

Para identificar os fatores que se associaram de forma independente à prevalência de anemia, os dados foram submetidos à análise de regressão logística (teste Wald). As variáveis com mais de duas categorias foram transformadas em variáveis "dummy". Foram incluídas no modelo todas as variáveis independentes estudadas, exceto a "renda familiar" por ter apresentado alta correlação com a "renda per capita" ( $\mathrm{r}>0,7)$, eliminando-se, assim, o problema da multicolinearidade. Adotou-se o procedimento "Enter" e consideraram-se associações estatisticamente significativas apenas aquelas que atingiram um $p<0,05$.

O projeto foi aprovado pelo Comitê de Ética em Pesquisa da Universidade Federal de Alagoas (Processo n. ${ }^{\circ}$ 010102/03-55) e os dados foram coletados após autorização dos pais, explicitada por meio da assinatura do Termo de Consentimento Livre e Esclarecido.

\section{Resultados}

Das 702 crianças selecionadas para o estudo, 15 não estavam presentes no domicílio e em 21 não se obteve autorização dos pais para participação. Assim, a amostra final foi de 666 indivíduos, representando $89,1 \%$ do tamanho da amostra calculado.

A prevalência de anemia foi de $45,0 \%$ e o valor médio de hemoglobina foi $11,0 \pm 1,53 \mathrm{~g} / \mathrm{dL}$. Um percentual de $34,4 \%$ das crianças apresentava anemia leve, 9,5\% anemia moderada e 1,2\% tinha quadro de anemia grave. Não foram observadas diferenças estatisticamente significantes da prevalência de anemia $(p=0,87)$ ou médias de $\mathrm{Hb}$ $(p=0,64)$ entre meninos $(\mathrm{n}=317 ; 45,4 \% ; 10,9 \mathrm{~g} / \mathrm{dL} \pm$ $1,57)$ e meninas $(\mathrm{n}=349 ; 44,7 \% ; 11,0 \mathrm{~g} / \mathrm{dL} \pm 1,49)$.

Quanto à faixa etária, observou-se um aumento do nível de $\mathrm{Hb}$ em função da idade $(\mathrm{r}=0,44$; $p<0,001)$. As crianças anêmicas apresentaram menor média de idade que as não anêmicas. A prevalência 
de anemia mais elevada foi observada nas crianças de 6 a 12 meses e a menor nas maiores de 36 meses (Tabela 1). Estratificando a amostra em dois grupos etários: $\leq 24$ meses e $>24$ meses, constatou-se que a chance de anemia no primeiro grupo foi mais do que cinco vezes em relação a do segundo grupo.

Um percentual de $69,5 \%$ das crianças residia na área urbana, sendo as residentes na área rural as que apresentaram a maior prevalência de anemia $(p=0,003)$, conforme indicado na Tabela 1 . Morar em municípios do interior, desde que na zona urbana, não aumentou a prevalência de anemia em relação à observada em crianças de Maceió (40,4\% vs. 39,8\%, respectivamente; $p>0,05)$. Contudo, residir em áreas rurais desses municípios elevou significativamente a sua ocorrência $(56,2 \% ; p=0,001)$. A falta de esgota- mento sanitário, água encanada e um menor número de cômodos no domicílio foram outras variáveis que se constituíram em fatores de risco para a anemia $(p<0,05)$.

A renda familiar mediana foi de $\mathrm{R} \$ 315,00$ (trezentos e quinze reais). A maioria das famílias que compunham o decil superior de renda da amostra ganhava apenas cerca de três salários mínimos, evidenciando o baixo nível econômico dessa população. O salário mínimo vigente no Brasil em janeiro de 2006 era de R\$300,00 (trezentos reais) correspondendo a, aproximadamente, US\$ 133,30 dólares $(\mathrm{US} \$ 1,00=\mathrm{R} \$ 2,25)$.

Todos os indicadores socioeconômicos investigados (renda familiar total ou per capita e posse de itens de consumo) apresentaram associação com a

Tabela 1

Prevalência de anemia em crianças menores de cinco anos, segundo variáveis ambientais. Alagoas, Brasil, 2006.

\begin{tabular}{|c|c|c|c|c|}
\hline \multirow{2}{*}{ Variáveis } & \multirow{2}{*}{$\mathbf{N}$} & \multicolumn{2}{|c|}{ Anemia** } & \multirow{2}{*}{$\begin{array}{l}\text { Razão de chances } \\
(\text { IC } 95 \%)^{\star * *}\end{array}$} \\
\hline & & $\mathrm{n}$ & $\%$ & \\
\hline \multicolumn{5}{|l|}{ Faixa etária (meses) } \\
\hline $6-12$ & 117 & 88 & 75,2 & $13,89 *(6,50-27,91)$ \\
\hline $13-24$ & 182 & 113 & 62,1 & $7,23^{*}(3,80-13,89)$ \\
\hline $25-36$ & 151 & 61 & 40,4 & $2,99 *(1,55-5,83)$ \\
\hline $37-48$ & 124 & 21 & 16,9 & $0,90(0,42-1,93)$ \\
\hline $49-60$ & 92 & 17 & 18,5 & 1 \\
\hline Total & 666 & 300 & 45,0 & - \\
\hline \multicolumn{5}{|l|}{ Tipo de esgotamento sanitário } \\
\hline Sanitário ligado à rede de esgoto & 112 & 45 & 40,2 & 1 \\
\hline Sanitário ligado à fossa & 422 & 172 & 40,7 & $1,02(0,67-1,57)$ \\
\hline Sem instalação sanitária ou outro & 130 & 82 & 63,1 & $2,54 *(1,51-4,27)$ \\
\hline \multicolumn{5}{|l|}{ Água encanada } \\
\hline Sim, dentro de casa & 432 & 166 & 38,4 & 1 \\
\hline Sim, no quintal & 77 & 44 & 57,1 & $1,02(0,67-1,57)$ \\
\hline Não & 157 & 90 & 57,3 & $2,54^{*}(1,51-4,27)$ \\
\hline \multicolumn{5}{|c|}{ Material predominante na construção da residência } \\
\hline Casa de tijolo ou edifício & 587 & 257 & 43,8 & 1 \\
\hline Diferente de alvenaria & 77 & 41 & 53,2 & $1,46(0,91-2,35)$ \\
\hline \multicolumn{5}{|l|}{ Água usada para beber } \\
\hline Rede pública ou água mineral & 449 & 185 & 41,2 & 1 \\
\hline Rio, poço, nascente, chuva & 217 & 115 & 53,0 & $1,61^{*}(1,16-2,23)$ \\
\hline \multicolumn{5}{|l|}{ Número de compartimentos da casa } \\
\hline$\leq 4$ cômodos & 266 & 136 & 51,1 & $1,50 *(1,10-2,06)$ \\
\hline$>4$ cômodos & 400 & 164 & 41,0 & 1 \\
\hline \multicolumn{5}{|l|}{ Situação do domicílio } \\
\hline Rural & 203 & 114 & 56,1 & $1,90 *(1,36-2,65)$ \\
\hline Urbano & 462 & 186 & 40,3 & 1 \\
\hline
\end{tabular}

${ }^{*}$ Indicam diferenças $(p<0,05)$ pelo teste $\chi^{2} ;{ }^{* *}$ Anemia $=$ hemoglobina $<11 \mathrm{~g} / \mathrm{dL} ;{ }^{* *} \mathrm{IC} 95 \%=$ Intervalo de confiança a $95 \%$. 
prevalência de anemia $(p<0,01)$, o mesmo ocorrendo em relação à escolaridade materna. Um maior número de membros na família também se constituiu em fator de risco importante (Tabela 2).

Quanto às condições de saúde, constatou-se que a anemia esteve associada à maior frequência de diarréia ou tosse (Tabela 3). Cerca de 90\% das crianças não tinham frequentado creches nos últimos 12 meses, tendo-se observado menor prevalência de anemia entre as crianças que haviam frequentado tais estabelecimentos. Mais de $28 \%$ das crianças não haviam recebido a suplementação da vitamina $\mathrm{A}$, sendo essas crianças as que apresentaram maior prevalência de anemia, independentemente do tempo decorrido desde o recebimento da dose de vitamina A. Apenas $10,5 \%$ das crianças tinham acesso a serviços de saúde particulares. Nestas, a frequência de anemia foi significativamente inferior à observada nas crianças assistidas por serviços públicos de saúde. Cerca de $14 \%$ das crianças tinham histórico de internação hospitalar nos últimos 12 meses, fato que também se constituiu em fator de risco para anemia (Tabela 3 ).

Em relação aos índices antropométricos estudados, não foram constatadas diferenças estatisticamente significantes entre as médias de hemoglobina, bem como entre as prevalências de anemia nas crianças com déficit $(Z<-2)$ em relação às eutróficas.

O peso médio ao nascer foi de $3279 \mathrm{~g} \pm 0,56$, sendo a prevalência de baixo peso $(<2500 \mathrm{~g})$ de $5,2 \%$ $(n=52)$. As crianças que nasceram com peso insufi-

Tabela 2

Prevalência de anemia em crianças menores de cinco anos, segundo variáveis socioeconômicas. Alagoas, Brasil, 2006

\begin{tabular}{|c|c|c|c|c|}
\hline \multirow{2}{*}{ Variáveis } & \multirow{2}{*}{$\mathbf{N}$} & \multicolumn{2}{|c|}{ Anemia** } & \multirow{2}{*}{$\begin{array}{l}\text { Razão de chances } \\
\qquad(\text { IC95\%)*** }\end{array}$} \\
\hline & & $\mathrm{n}$ & $\%$ & \\
\hline \multicolumn{5}{|c|}{$\begin{array}{l}\text { Posse dos itens: televisão, fogão a gás, geladeira, } \\
\text { máquina de lavar e automóvel }\end{array}$} \\
\hline$<3$ itens & 233 & 139 & 59,7 & $3,07 *(1,81-5,20)$ \\
\hline 3 itens & 350 & 134 & 38,3 & $1,29(0,77-2,14)$ \\
\hline$>3$ itens & 83 & 27 & 32,5 & 1 \\
\hline \multicolumn{5}{|l|}{ Renda per capita (em US\$)**** } \\
\hline $1^{\circ}$ tercil: $<22.22$ & 184 & 98 & 53,3 & $1,84^{*}(1,21-2,80)$ \\
\hline $2^{\circ}$ tercil: 22.22 a 44.44 & 266 & 111 & 41,7 & $1,15(0,78-1,70)$ \\
\hline $3^{\circ}$ tercil: $>44.44$ & 175 & 67 & 38,3 & 1 \\
\hline \multicolumn{5}{|l|}{ Renda familiar (em US\$)**** } \\
\hline $1^{\circ}$ tercil: $<13333$ & 208 & 108 & 51,9 & $1,94 *(1,31-2,88)$ \\
\hline $2^{\circ}$ tercil: 13333 a 20444 & 215 & 97 & 45,1 & $1,47^{*}(1,00-2,18)$ \\
\hline $3^{\circ}$ tercil: > 20444 & 204 & 73 & 35,8 & \\
\hline \multicolumn{5}{|c|}{ Número de anos de estudo materno } \\
\hline$<5$ anos & 281 & 145 & 51,6 & $1,79 *(1,22-2,62)$ \\
\hline 5 a 8 anos & 160 & 74 & 46,2 & $1,56(0,99-2,46)$ \\
\hline$>8$ anos & 149 & 53 & 35,6 & 1 \\
\hline \multirow{2}{*}{\multicolumn{5}{|c|}{$\begin{array}{l}\text { Inscrição em programa público de redistribuição } \\
\text { de renda }\end{array}$}} \\
\hline & & & & \\
\hline Sim & 208 & 101 & 48,6 & $1,23(0,89-1,71)$ \\
\hline Não & 454 & 197 & 43,4 & 1 \\
\hline \multicolumn{5}{|c|}{ Número de membros da família } \\
\hline$<5$ pessoas & 294 & 118 & 40,1 & 1 \\
\hline$\geq 5$ pessoas & 370 & 182 & 49,2 & $1,44^{*}(1,05-1,97)$ \\
\hline
\end{tabular}

*Indicam diferenças $(p<0,05)$ pelo teste $\chi^{2} ;{ }^{* *}$ Anemia $=$ hemoglobina $<11 \mathrm{~g} / \mathrm{dL}$; ${ }^{* *} \mathrm{IC} 95 \%=$ Intervalo de confiança a $95 \%$; $* * *$ US\$1 $00=\mathrm{R} \$ 2.25$ em janeiro de 2006. 
ciente apresentaram prevalência de anemia mais elevada do que as nascidas com peso maior ou igual a $3000 \mathrm{~g}$, contudo tais diferenças atingiram significância apenas marginal $(0,05 \leq p<0,1)$.

Uma maior prevalência de anemia foi observada nas crianças cujas mães apresentassem uma das seguintes variáveis: idade em que teve o $1^{\circ}$ filho igual ou inferior a 20 anos, baixa estatura e sobrepeso (Tabela 4).
$\mathrm{Na}$ análise multivariada, as variáveis que permaneceram estatisticamente associadas à anemia foram idade $\leq 36$ meses $(p<0,001)$ e domicílio com cinco ou mais pessoas $(p=0,031)$ (Tabela 5$)$. Essas duas condições aumentaram as chances de anemia em, respectivamente, 7,29 e 1,92 vezes. As variáveis incluídas no modelo final da regressão logística classificaram corretamente $68,9 \%$ dos indivíduos anêmicos.

Tabela 3

Prevalência de anemia em crianças menores de cinco anos, segundo variáveis relacionadas às condições de saúde e de utilização dos serviços públicos. Alagoas, Brasil, 2006.

\begin{tabular}{|c|c|c|c|c|}
\hline \multirow{2}{*}{ Variáveis } & \multirow{2}{*}{$\mathbf{N}$} & \multicolumn{2}{|c|}{ Anemia** } & \multirow{2}{*}{$\begin{array}{l}\text { Razão de chances } \\
\left(\text { IC95\%) }{ }^{* * *}\right.\end{array}$} \\
\hline & & $\mathrm{n}$ & $\%$ & \\
\hline \multicolumn{5}{|c|}{ Diarréia nos últimos 15 dias } \\
\hline Sim & 167 & 103 & 61,7 & $2,47 *(1,72-3,54)$ \\
\hline Não & 499 & 197 & 39,5 & 1 \\
\hline \multicolumn{5}{|c|}{ Serviço de saúde usado pela criança quando doente } \\
\hline Serviço público & 581 & 276 & 47,5 & $1,89 *(1,11-3,23)$ \\
\hline Particular & 68 & 22 & 32,3 & 1 \\
\hline \multicolumn{5}{|c|}{ Tosse na última semana } \\
\hline Sim & 301 & 144 & 47,8 & $1,23(0,90-1,67)$ \\
\hline Não & 365 & 156 & 42,7 & 1 \\
\hline \multicolumn{5}{|c|}{ Internamento nos últimos 12 meses } \\
\hline Sim & 91 & 53 & 58,2 & $1,84 *(1,17-2,88)$ \\
\hline Não & 570 & 246 & 43,2 & 1 \\
\hline \multicolumn{5}{|c|}{ Realização de consulta pré-natal } \\
\hline Sim & 556 & 258 & 46,4 & 1 \\
\hline Não & 34 & 21 & 61,8 & $1,87(0,92-3,80)$ \\
\hline \multicolumn{5}{|c|}{ Recebeu suplementação de vitamina A nos últimos } \\
\hline \multicolumn{5}{|l|}{12 meses } \\
\hline Sim & 474 & 197 & 41,6 & 1 \\
\hline Não & 192 & 103 & 53,6 & $1,63 *(1,16-2,28)$ \\
\hline \multicolumn{5}{|c|}{ Frequentou creche no último ano } \\
\hline Sim & 76 & 16 & 21,0 & 1 \\
\hline Não & 590 & 284 & 48,1 & $3,48 *(1,96-6,18)$ \\
\hline
\end{tabular}

${ }^{*}$ Indicam diferenças $(p<0,05)$ pelo teste do $\chi^{2} ;{ }^{* *}$ Anemia $=$ hemoglobina $<11 \mathrm{~g} / \mathrm{dL} ;{ }^{* * *} \mid \mathrm{C} 95 \%=$ Intervalo de confiança a $95 \%$. 
Prevalência de anemia em crianças menores de cinco anos, segundo indicadores antropométricos da criança e da mãe e idade quando da ocorrência da menarca e nascimento do $1^{\circ}$ filho. Alagoas, Brasil, 2006.

\begin{tabular}{|c|c|c|c|c|}
\hline \multirow{2}{*}{ Variáveis } & \multirow{2}{*}{$\mathbf{N}$} & \multicolumn{2}{|c|}{ Anemia** } & \multirow{2}{*}{$\begin{array}{l}\text { Razão de chances } \\
(\text { IC95\%)*** }\end{array}$} \\
\hline & & $\mathrm{n}$ & $\%$ & \\
\hline \multicolumn{5}{|l|}{ Estatura-para-idade da criança } \\
\hline Não déficit $(Z \geq-2)$ & 570 & 253 & 44,4 & 1 \\
\hline Déficit $(Z<-2)$ & 85 & 41 & 48,2 & $1,17(0,74-1,84)$ \\
\hline \multicolumn{5}{|l|}{ Peso-para-idade da criança } \\
\hline Não déficit $(Z \geq-2)$ & 637 & 288 & 45,2 & 1 \\
\hline Déficit $(Z<-2)$ & 26 & 11 & 42,3 & $0,89(0,40-1,96)$ \\
\hline \multicolumn{5}{|l|}{ Peso-para-estatura da criança } \\
\hline Não déficit $(Z \geq-2)$ & 645 & 289 & 44,8 & 1 \\
\hline Déficit $(Z<-2)$ & 8 & 4 & 50,0 & $1,23(0,30-4,97)$ \\
\hline \multicolumn{5}{|l|}{ Peso ao nascer $(\mathrm{g})$} \\
\hline$\geq 3000$ (Normal) & 453 & 193 & 42,6 & 1 \\
\hline < 3000 (Baixo ou insuficiente) & 151 & 75 & 49,7 & $1,33(0,92-1,92)$ \\
\hline \multicolumn{5}{|c|}{ Idade em que a mãe teve o $1^{\circ}$ filho (anos) } \\
\hline$\leq 20$ & 373 & 186 & 49,9 & $1,45^{*}(1,01-2,06)$ \\
\hline 21 a 30 & 189 & 77 & 40,7 & 1 \\
\hline$>30$ & 27 & 8 & 29,6 & $0,61(0,25-1,47)$ \\
\hline \multicolumn{5}{|l|}{ Estatura materna $(\mathrm{cm})$} \\
\hline$<152,2\left(1^{\circ}\right.$ quartil) & 118 & 64 & 54,2 & $2,34 *(1,38-3,96)$ \\
\hline 152,2 a 155,7 ( $2^{\circ}$ quartil) & 122 & 55 & 45,1 & $1,62(0,96-2,73)$ \\
\hline 155,8 a 159,5 (3 quartil) & 115 & 53 & 46,1 & $1,69(0,99-2,86)$ \\
\hline$>159,5$ (4º quartil) & 119 & 40 & 33,6 & 1 \\
\hline \multicolumn{5}{|c|}{ Índice de Massa Corporal materno (kg/m²) } \\
\hline$<18,5$ (Baixo peso) & 27 & 12 & 44,4 & $0,79(0,35-1,75)$ \\
\hline 18,5 a 24,9 (Eutrofia) & 224 & 113 & 50,4 & 1 \\
\hline 25 a 29,9 (Sobrepeso) & 148 & 58 & 39,2 & $0,63^{*}(0,41-0,96)$ \\
\hline$\geq 30,0$ (Obesidade) & 73 & 28 & 38,4 & $0,61(0,36-1,05)$ \\
\hline \multicolumn{5}{|l|}{ Idade da Menarca (anos) } \\
\hline$<12\left(1^{\circ}\right.$ tercil $)$ & 71 & 39 & 54,9 & $1,68(0,99-2,87)$ \\
\hline 12 a $13\left(2^{\circ}\right.$ tercil $)$ & 238 & 100 & 42,0 & 1 \\
\hline$>13\left(3^{\circ}\right.$ tercil $)$ & 151 & 68 & 45,0 & $1,13(0,75-1,71)$ \\
\hline
\end{tabular}

${ }^{*}$ Indicam diferenças $(p<0,05)$ pelo teste do $\chi^{2} ;{ }^{* *}$ Anemia $=$ hemoglobina $<11 \mathrm{~g} / \mathrm{dL} ;{ }^{* * \star} I \mathrm{C} 95 \%=$ Intervalo de confiança a $95 \%$.

Tabela 5

Variáveis associadas à prevalência de anemia após análise multivariada e suas respectivas medidas de associação.

\begin{tabular}{|c|c|c|}
\hline \multirow{2}{*}{ Variáveis } & \multicolumn{2}{|c|}{ Prevalência de anemia } \\
\hline & Razão de chances (IC95\%)*** & $p^{*}$ \\
\hline Idade da criança $\leq 36$ meses & $7,29(3,67-14,46)$ & $<0,001 * *$ \\
\hline Família com cinco ou mais membros & $1,92(1,06-3,49)$ & 0,031 ** \\
\hline Menor estatura materna (< 152,2 cm $-1^{\circ}$ quartil) & $2,19(0,98-4,90)$ & 0,057 \\
\hline Residir em domicílio que não seja de alvenaria & $2,61(0,97-7,00)$ & 0,057 \\
\hline Idade em que a mãe teve o $1^{\circ}$ filho $\leq 20$ anos & $1,86(0,97-3,57)$ & 0,061 \\
\hline Frequentou creche no último ano & $0,42(0,16-1,07)$ & 0,068 \\
\hline Peso ao nascer baixo ou insuficiente & $1,77(0,93-3,36)$ & 0,080 \\
\hline Mãe portadora de sobrepeso (25 a $29,9 \mathrm{~kg} / \mathrm{m}^{2}$ ) & $0,60(0,33-1,09)$ & 0,095 \\
\hline
\end{tabular}

* Regressão Logística (teste de Wald); ** $p<0,05 ;{ }^{* * *} \mathrm{IC} 95 \%=$ Intervalo de confiança a $95 \%$. 


\section{Discussão}

A prevalência de anemia encontrada, de 45,0\%, superou em cinco pontos percentuais os níveis considerados pela Organização Mundial da Saúde (OMS) como grave problema do ponto de vista epidemiológico. ${ }^{16}$ Esse percentual de anemia foi cerca de duas vezes superior aos recentemente divulgados para o Brasil, de $20,9 \%$, e para a Região Nordeste, de 25,5\% na Pesquisa Nacional de Demografia e Saúde da Criança e da Mulher, 17 em 2006, mesmo ano da realização do presente estudo. Níveis igualmente elevados foram encontrados em Pernambuco $(46,9 \%)^{18}$ e na Paraíba $(36,4 \%),{ }^{19}$ a partir de levantamentos realizados em amostras probabilísticas representativas dos pré-escolares dos respectivos Estados.

Concordando com dados de um levantamento realizado na zona da mata do Estado de Pernambuco, não se constatou diferenças nas prevalências de anemia entre meninos e meninas na população estudada. 20 Segundo Lima et al.,20 o sexo não constitui um fator de risco de anemia em pré-escolares, havendo maior risco de anemia no sexo feminino a partir da menarca.

$\mathrm{Na}$ análise de regressão múltipla, a variável mais fortemente associada à prevalência de anemia foi idade inferior a 36 meses, tendo o risco de anemia sido maior do que sete vezes nessa faixa etária em relação às com idade entre 49 e 60 meses. Embora se reconheça que a utilização da razão de chances obtida por regressão logística possa superestimar o efeito de determinadas variáveis sobre desfechos com prevalências superiores a 10\%,21 os dados da literatura são consistentes em apontar a força dessa associação.22-24 Isto pode ser explicado pela maior velocidade de crescimento nessa faixa etária, alta frequência de desmame precoce, pelo atraso na introdução de alimentos ricos em ferro na dieta da criança e pela maior prevalência de doenças como a diarréia e infecções respiratórias. ${ }^{23}$ Nas crianças com idades superiores a 35 meses a velocidade de crescimento torna-se mais lenta, ocorre um menor consumo de leite de vaca (o qual diminui a biodisponibilidade do ferro no trato digestivo) e maior ingestão de alimentos fontes de ferro que sejam do hábito familiar. ${ }^{25}$

Estudo realizado em Pernambuco 12 encontrou maior prevalência de anemia entre as crianças das áreas rurais em comparação às residentes na área metropolitana do Recife. Esse diferencial seria justificado, segundo os autores, pela menor infraestrutura de serviços públicos na área rural, determinando pouco acesso a serviços de saúde, saneamento básico, água potável, renda, oportunidade de emprego, informação e educação. Na presente investigação residir em área rural perdeu significância como fator de risco para anemia após a análise multivariada. Possivelmente, em Alagoas, as condições de vida prevalecentes nas áreas urbanas não sejam tão diferentes daquelas observadas nas áreas rurais, o que explicaria esse resultado.

Concordando com os resultados de outros estudos, 18,22,26 observou-se que maior número de membros na família se associou à maior prevalência de anemia, provavelmente devido à fatores relacionado à menor renda per capita e, consequentemente, ao menor poder aquisitivo dessas famílias. Em trabalho realizado por Assunção et al. ${ }^{22} \mathrm{em}$ menores de cinco anos, apenas a idade da criança e a renda familiar permaneceram como variáveis associadas à anemia após análise multivaridada. Maiores níveis de renda familiar associam-se positivamente com a concentração da $\mathrm{Hb}$ e negativamente com a prevalência de anemia, o mesmo ocorrendo em relação à escolaridade materna, tal como observado por Oliveira et al.12 Segundo esses autores, a escolaridade do chefe da casa e/ou responsável pela criança presume uma utilização mais eficiente da renda e dos serviços públicos disponíveis. Assis et al.25 acrescentam que contar com mais de um pré-escolar no domicílio contribuiria para maior dificuldade da família em prestar atenção adequada a essas crianças, sobretudo nos estratos de menor nível socioeconômico.

A desnutrição infantil, quando avaliada pela antropometria, se manifesta por meio do baixo peso ao nascer $(<2500 \mathrm{~g})$ e do déficit de crescimento (ponderal e/ou estatural). De acordo com Batista Filho e Rissin, 27 a assistência pré-natal adequada constitui uma medida efetiva de prevenção do baixo peso ao nascer, que agiria reduzindo o risco de deficiência de $\mathrm{Hb}$ nos primeiros meses de vida. Em estudos populacionais, o baixo peso ao nascer e a anemia estão quase sempre relacionados, pois o primeiro é fator importante na determinação do segundo. Além disso, as reservas de ferro ao nascer são proporcionais ao peso da criança, ou seja, menor peso ao nascimento representa menor reserva de ferro e maior risco para anemia. 25 No presente estudo, essa associação não atingiu significância estatística $(p=0,08)$ após análise de regressão logística.

Conforme já referido, as crianças que não haviam recebido a suplementação da vitamina A apresentaram maior prevalência de anemia. A suplementação com ácido fólico, vitamina $\mathrm{C}$ e/ou vitamina A tem sido recomendada em regiões onde se 
supõe que essas deficiências contribuam para a ocorrência de anemia. 12 A deficiência de vitamina $\mathrm{A}$ representa problema de saúde pública para diversos países, incluindo o Brasil, especialmente na Região Nordeste, e pode levar à anemia, sendo de ocorrência relativamente comum em pré-escolares, corroborando a hipótese de que deficiências de micronutrientes ocorrem na forma de múltiplas carências, sobretudo as de ferro e de vitamina A. Lynch 28 demonstrou que a deficiência sérica de retinol afeta o transporte de ferro e resulta em uma condição com características de anemia ferropriva que só responde ao tratamento com ferro após suplementação de vitamina A. Desse modo, a suplementação de vitamina A pode se constituir em importante medida para a adequada eficácia no combate à anemia em regiões endêmicas. Estudo realizado na região semiárida de Alagoas revelou que $44,8 \%$ das crianças tinham hipovitaminose A. 29

Além da indisponibilidade de dados sobre consumo alimentar das crianças, este estudo apresenta como limitação a ausência de outros indicadores hematimétricos e bioquímicos como ferritina, capacidade de ligação do ferro, protoporfirina eritrocitária, entre outros, para caracterizar a provável etiologia do problema, embora haja

\section{Referências}

1. World Health Organization/Centers for Disease Control and Prevention. Assessing the iron status of populations: a report of a joint World Health Organization/Centers for Disease Control technical consultation on the assessment of iron status at the population level. [acesso em 11 mai 2009] Disponível em: http://whqlibdoc.who.int/publications/ 2004/9241593156_eng.pdf

2. World Health Organization. Iron deficiency anaemia. Assessment, prevention and control. A guide for programme managers. Geneva; 2001.

3. Stoltzfus RJ. Iron deficiency: global prevalence and consequences. Food Nutr Bull. 2003; 24 (Suppl 4): S99-103.

4. Batista Filho M. O controle das anemias no Brasil. Rev Bras Saúde Mater Infant. 2004; 4: 121-3.

5. Spinelli MGN, Marchioni DML, Souza JMP, Souza SB, Szarfarc SC. Fatores de risco para anemia em crianças de 6 a 12 meses no Brasil. Rev Panam Salud Publica. 2005; 17 : 84-91.

6. Monteiro CA, Szarfarc SC, Mondini L. Tendência secular da anemia na infância na cidade de São Paulo (1984-1996). Rev Saúde Pública. 2000; 34: 62-72.

7. Monteiro CA. A dimensão da pobreza, da fome e da desnutrição no Brasil. Estud Av. 1995; 24: 195-207.

8. Santos CD, Santos LMP, Figueiroa JN, Marroquim PMG, Oliveira MAA. Anemia em escolares da primeira série do consenso que prevalências maiores que $40 \%$ têm como causa mais provável a deficiência de ferro dietético. ${ }^{12}$ Outra limitação provém do seu desenho metodológico (transversal), o que interfere na interpretação dos resultados na medida em que não é possível identificar se os fatores de risco foram antecedentes à anemia. Apesar desses óbices, esse estudo apresenta especial relevância por ter sido realizado com amostra adequada, representativa das crianças de um estado brasileiro, utilizando indicador epidemiologicamente válido para diagnóstico de anemia e, assim, disponibilizando informação útil para ações de planejamento do setor público, bem como constituindo um "base-line" para futuros estudos de tendência secular e/ou de avaliação de impacto de programas.

Os resultados apresentados permitem concluir que a anemia representa um importante problema de saúde pública em Alagoas, afetando proporção relevante de crianças, sobretudo, aquelas menores de 37 meses e que pertencem a famílias com cinco ou mais indivíduos. Não somente as precárias condições socioeconômicas explicam esses resultados, mas também a deficiência na assistência à saúde, características que devem nortear as políticas públicas de prevenção e controle. ensino fundamental da rede pública de Maceió, Alagoas, Brasil. Cad Saúde Pública. 2002; 18: 1757-63.

9. Ferreira HS, Assunção ML, Vasconcelos VS, Melo FP, Oliveira CG, Santos TO. Saúde de populações marginalizadas: desnutrição, anemia e enteroparasitoses em crianças de uma favela do "Movimento dos Sem Teto", Maceió, Alagoas. Rev Bras Saúde Matern Infant. 2002; 2 : 177-85.

10. IBGE (Instituto Brasileiro de Geografia e Estatística). Pesquisa Nacional por Amostra de Domicílios: Síntese de Indicadores - 2004. Rio de Janeiro: IBGE, 2005.

11. Urani A. Um diagnóstico socioeconômico do Estado de Alagoas a partir de uma leitura dos dados da Pesquisa Nacional por Amostra de Domicílios do IBGE (1992-2004). Instituto de Estudos do Trabalho e Sociedade. 15p. 2005.

12. Oliveira MAA, Osório MM, Raposo, MCF. Concentração de hemoglobina e anemia em crianças no Estado de Pernambuco, Brasil: fatores socioeconômicos e de consumo alimentar associados. Cad Saúde Pública. 2006; 22: 216978.

13. Paiva AA, Rondo PHC, Silva SSB, Latorre MRDO. Comparison between the HemoCue ${ }^{\circledR}$ and an automated counter for measuring hemoglobin. Rev Saúde Pública. 2004; 38: 585-7. 
14. De Maeyer EM, Dallman P, Gurney JM, Hallberg L, Sood SK, Srikantia SG. Prévenir et combattre l'anémie ferriprive dans le cadre des soins de santé primaires. Génève: OMS; 1991.

15. César CLG, Figueiredo GM, Westphal MF, Cardoso MRA, Costa MZA, Gattás VL. Morbidade referida e utilização de serviços de saúde em localidades urbanas brasileiras: Metodologia. Rev Saúde Pública. 1996; 30: 153-60.

16. Batista-Filho M, Souza AI, Bresani CC. Anemia como problema de saúde pública - uma realidade atual. Ciênc Saúde Coletiva. 2008; 13: 1917-22.

17. Ministério da Saúde. Pesquisa Nacional de Demografia e Saúde da Criança e da Mulher. Anemia e Hipovitaminose A. [acesso em 11 mai 2009] Disponível em: http://bvsms.saude.gov.br/bvs/pnds/anemia.php

18. Silva SCL, Batista Filho M, Miglioli TC. Prevalência e fatores de risco de anemia em mães e filhos no Estado de Pernambuco. Rev Bras Epidemiol. 2008; 11: 266-77.

19. Oliveira RS, Diniz AS, Benigna MJC, Miranda-Silva SM, Lola MM, Gonçalves MC, Asciutti-Moura L, Rivera MA, Santos LMP. Magnitude, distribuição espacial e tendência da anemia em pré-escolares da Paraíba. Rev Saúde Pública. 2002; 36: 26-32.

20. Lima ACVMS, Lira PIC, Romani SAM, Eickmann SH, Piscoya MD, Lima MC. Fatores determinantes dos níveis de hemoglobina em crianças aos 12 meses de vida na Zona da Mata Meridional de Pernambuco. Rev Bras Saúde Mater Infant. 2004; 4: 35-43.

21. Francisco PMSB, Donalisio MR, Barros MBA, Cesar CLG, Carandina L, Goldbaum K. Medidas de associação em estudo transversal com delineamento complexo: razão de chances e razão de prevalência. Rev Bras Epidemiol. 2008; 11: 347-55.

Recebido em 5 de janeiro de 2009

Versão final apresentada em 28 de setembro de 2009

Aprovado em 21 de outubro de 2009
22. Assunção MCF, Santos IS, Barros AJD, Gigante DP, Victora CG. Anemia em menores de seis anos: estudo de base populacional em Pelotas, RS. Rev Saúde Pública. 2007; 41: 328-35.

23. Silva LSM, Giugliani ERJ, Aerts DRGC. Prevalência e determinantes de anemia em crianças de Porto Alegre, RS, Brasil. Rev Saúde Pública. 2001; 35: 66-73.

24. Vieira ACF, Diniz AS, Cabral PC, Oliveira RS, Lóla MMF, Silva SMM, Kolsteren P. Avaliação do estado nutricional de ferro e anemia em crianças menores de 5 anos de creches públicas. J Pediatr. 2007; 83: 370-6.

25. Assis AMO, Barreto ML, Santos NS, Oliveira LPM, Santos SMC, Pinheiro SMC. Desigualdade, pobreza e condições de saúde e nutrição na infância no Nordeste brasileiro. Cad Saúde Pública. 2007; 23: 2337-50.

26. Neuman NA, Tanaka OY, Szarfarc SC, Guimarães PRV, Victora CG. Prevalência e fatores de risco para anemia no Sul do Brasil. Rev Saúde Pública. 2000; 34: 56-63.

27. Batista Filho M, Rissin A. Deficiências nutricionais: ações específicas do setor saúde para o seu controle. Cad Saúde Pública. 1993; 9: 130-5.

28. Lynch SR. Interaction of iron with other nutrients. Nutr Rev. 1997; 55: 102-10.

29. Vasconcelos AMA, Ferreira HS. Prevalência de hipovitaminose A em crianças da região semi-árida de Alagoas (Brasil), 2007. Arch Latinoam Nutr. 2009; 59: 152-8. 